

\section{polisemie}

\section{I}

\section{1}

Direzione

Stefano Milonia (University of Warwick)

Comitato scientifico

Giulia Bassi (Università degli Studi di Siena)

Mario Cianfoni (Sapienza Università di Roma)

Stefano Colangelo (Alma Mater Studiorum - Università degli Studi di Bologna)

Luigi Marinelli (Sapienza Università di Roma)

Carlo Pulsoni (Università degli Studi di Perugia)

Niccolò Scaffai (Università degli Studi di Siena)

Costantino Turchi (Sapienza Università di Roma)

Fabio Zinelli (École Pratique des Hautes Études)

\section{Redazione}

Stefano Bottero (Università Ca' Foscari Venezia)

Mattia Caponi (Sapienza Università di Roma)

Carlo Londero (Università degli Studi di Udine)

Giorgio Tranchida (Alma Mater Studiorum - Università degli Studi di Bologna)

Samuele Maria Visalli (Sapienza Università di Roma)

Arianna Saggio (Sapienza Università di Roma)

Andrea Bongiorno (Aix-Marseille Université)

Giulia Boitani (University of Cambridge)

Alessandra Frustaci

Polisemie è una rivista annuale pubblicata dalla University of Warwick Press.

Gli articoli pubblicati nella sezione Saggi sono sottoposti a double-blind peer review.

Licenza Creative Commons - Attribuzione (CC-BY 4.0).

ISSN: 2634-1867

DOI: 10.31273 /polisemie.v1

Immagine copertina: Serse Luigetti 


\title{
LE POESIE PIÙ RECENTI DI MILO DE ANGELIS
}

\author{
Patrycja Polanowska
}

Nell'antologia Poesia e Storia di Niva Lorenzini e Stefano Colangelo il periodo nella poesia italiana che va dal 1976 al 2010 viene esplicitamente definito come stagione del corpo. Un'immagine simbolo in cui si rispecchia quest'impostazione è quella del 1979, ossia del palco del Festival di Castel Porziano crollato sotto il peso del pubblico. ${ }^{1}$ L'avvenimento viene rappresentato persino come «un'allegoria del peso di un corpo e di un soggetto impossibili da contenere per una poesia che vuole essere autonoma, che nega risolutamente la forma e lo stile». ${ }^{2}$ Quest'approccio ci fa vedere allora come la presenza di una significativa componente della corporalità in un'esperienza poetica non deve coincidere automaticamente con una scissione che liberi la parola dall'egocentrismo lirico. Sembra, per l'appunto, la negazione dei valori classici collegata all'avanzamento della visione egotica a portare alla crisi del genere poetico. L'anno 1976, presentato all'apertura come un metaforico punto d'orientamento, è anche l'anno in cui viene pubblicata la raccolta d'esordio di Milo De Angelis, intitolata Somiglianze. Resta indiscutibile che soprattutto i suoi primi componimenti vengono segnati da un'ottica strettamente legata alla corporalità che si realizza a sua volta nella concretezza del qui-e-ora. Sarà proprio quest'aspetto a non permettere di etichettarlo come poeta neo-orfico, pur percependo l'inafferrabilità dei suoi versi, il costruirsi del discorso su «istantanee intermittenze emotive». ${ }^{3}$ È però necessario, prima di collocare quest'opera nella tendenza dominante, riconoscere la molteplicità dei modi in cui il corpo può inserirsi nello spazio della scrittura in versi.

\footnotetext{
${ }^{1}$ Cfr. Guido Mazzoni, Sulla storia sociale della poesia contemporanea in Italia, in «Ticontre. Teoria Testo Traduzione», 8 (2017), pp. 1-26. Per consultare la trascrizione integrale del festival, si veda Il romanzo di Castel Porziano: tre giorni di pace, amore e poesia, a cura di Simone Carella, Paola Febbraro, Simona Barberini, Viterbo, Stampa alternativa, 2015.

${ }^{2}$ Niva Lorenzini, Stefano Colangelo, Poesia e Storia, Milano, Bruno Mondadori, 2013, p. 271.

${ }^{3}$ Ivi, p. 273.
} 
La centralizzazione dell'immaginario poetico sulla figura del poeta-personaggio si mostra come risposta alla ricerca identitaria, alle indagini dell'essere nel mondo, che prendono questa forma attorno agli anni Sessanta e Settanta. «Il corpo», come leggiamo in Poesia e Storia, «è insomma lo strumento per snodare le esperienze dell'essere umano, le profondità dell'inconscio, e liberare in questo modo i flussi, le onde di desiderio in una società repressa e repressiva $\gg .{ }^{4}$ Questa linea, volendo opporsi agli sperimentalismi, ${ }^{5}$ ad un eccesso d'impegno, si rifugia nel dominio dell'io, senza possibilità di ricreare il vecchio codice lirico, essendo sbarrata dall'insicurezza e dalla dismisura che penetrano i versi e portano alla «disintegrazione del soggetto». ${ }^{6}$ Le due reazioni modello che nascono in risposta alla crisi del soggetto, distinte da Guido Mazzoni in riferimento alla svolta nella poesia moderna, cioè l'espressionismo e il crepuscolarismo sembrano ricondurci, mutatis mutandis, fino al periodo da noi esaminato e alle posizioni descritte da Gianluigi Simonetti, come il mito delle origini e la nevrosi della fine, ${ }^{7}$ dominanti nel quadro poetico degli anni Settanta e seguenti. Nella stagione del corpo la poesia si fonda allora su un'impostazione quasi schizofrenica: partecipe della disintegrazione del soggetto, cerca di mantenere la centralità dell'esperienza fortemente individuale. Contraddice così il proprio tentativo di farsi uno strato indipendente dell'esistere. La sua liberazione dell'atto poetico dal progresso temporale e il riconoscimento dell'autonomia del verso, che non teme un confronto con la tradizione, vengono frenate però dall'assolutizzazione dell'esperienza, quasi epifanica, dell'universale uomo. Invece di allargare le proprie capacità percettive - quando la «scoperta dell'alterità» ${ }^{8}$ può portare al superamento del dualismo tra contingente ed eterno - crea una linea di ermetismo rovesciato, ossia un ermetismo impossibile: confessionale, euforico ed irrazionale.

\footnotetext{
${ }^{4}$ Ivi, p. 270.

${ }^{5}$ Cfr. Niva Lorenzini, Da Postkarten a Cataletto: la messa in scena del corpo, in Corpo e poesia nel Novecento italiano, Milano, Bruno Mondadori, 2009, pp. 17-18, 102-111, ma anche Enrico Testa, A strappi e toppe. Il soggetto nella poesia di Sanguineti, in Costruzioni e decostruzioni dell'io lirico nella poesia italiana da Soffici a Sanguineti, a cura di Damiano Frasca, Caroline Lüderssen e Christine Ott, Firenze, Cesati, 2015, pp. 91-99. Nei saggi viene sottolineato come in Sanguineti il linguaggio pur seguendo l'esperienza del corpo traccia solo la sua separatezza dall'io.

${ }^{6}$ Guido Mazzoni, Sulla poesia moderna, Bologna, il Mulino, 2005, pp. 180-181; «Quando pensiamo allidealtipo della poesia romantica, pensiamo a una forma di individualismo sicuro $e$ misurato: sicuro di sé nel rivendicare il valore universale di un'esperienza personale; misurato nel bilanciare espressivismo e antiespressivismo, i diritti del talento individuale e il rispetto di alcune strutture del discorso pubblico, radicate nel senso comune o nella tradizione».

${ }^{7} \mathrm{Cfr}$. Gianluigi Simonetti, Mito delle origini, nevrosi della fine. Identità della poesia contemporanea, in La letteratura circostante. Narrativa e poesia nell'Italia contemporanea, Bologna, il Mulino, 2018, pp. 139-226.

${ }^{8}$ Lorenzini, Corpo e poesia nel Novecento italiano, p. 5.
} 
La scelta tra l'io lirico e il corpo, in quanto strumento della conoscenza, comporta modifiche anche nella percezione del tempo e dello spazio. ${ }^{9}$ L'approccio emerso nella seconda metà degli anni Settanta, pur definito come stagione del corpo, non riesce a riprendere la linea che va da Campana a Rosselli, da Rebora a Zanzotto, dove il corpo immedesimato nelle parole estende le possibilità del linguaggio e rende più piena la percezione dello spazio e del tempo. Gli anni del mito delle origini, che sono anni dominati dalla diffusione della pratica della performance, si esauriscono invece in un tempo eternamente presente e nella rappresentazione mimetica e diretta dell'accadere. ${ }^{10}$ Insieme alla caduta della verticalità lirica, cade qui anche il senso della durata, delimitando lo spazio poetico alla figura dell'io e alla sua individuale percezione del mondo. A tale proposito Niva Lorenzini osserva:

$[\mathrm{L}] \mathrm{a}$ dilatazione delle possibilità di esperienza rende infatti da una parte definitivamente improponibile il concetto di durata temporale (sostituito semmai dalla densità degli istanti), dall'altra enfatizza la dimensione di un presente su cui si tesse senza sosta una trama di frammenti variamente combinati, sostituibili in eterno in quanto perfettamente omogenei [...]. Si agisce sull'oggi, ontologizzato e astorico, abolite le contraddizioni e insieme le categorie di passato e futuro. ${ }^{11}$

Il concetto del puro presente, sottratto al rapporto con passato e futuro, provoca un appiattimento del percorso poetico, cui viene negata qualsiasi tensione al sublime, ma al tempo stesso, senza stabilire un aggancio vitale tra istanti, viene ridotta anche la prospettiva orizzontale, fino a far finire le immagini nella condizione di un'immobilità profonda, provocata dalla focalizzazione dello sguardo poetico su punti individuali, ma indifferenziati. Ritroviamo un'osservazione correlata in Alfonso Berardinelli, quando il critico afferma: «Noi non riusciamo più né a relegare del tutto la poesia in un ambito di perfetta autonomia estetica, indifferente alla storia, né a trovare un rapporto credibile e dinamico fra poesia e storia, sotto il segno dell'utopia e del progresso $\gg .{ }^{12}$ Lo sviluppo dialettico della produzione artistica, sospesa tra assoluto e storia, si esaurisce nell'imperativo dell'incondizionata e idiosincratica riproduzione del reale.

\footnotetext{
${ }^{9}$ Cfr. Ivi, pp. 9-13.

${ }^{10}$ Cfr. Pier Vittorio Tondelli, Un weekend postmoderno: cronache dagli anni Ottanta, Milano, Bompiani, 1990; Andrea Zanzotto, Diffidare, gola, corpo, movimenti, teatro in Fosfeni, Milano, Mondadori, 1983.

${ }^{11}$ Niva Lorenzini, Il presente della poesia, Bologna, il Mulino, 1991, p. 16.

${ }^{12}$ Alfonso Berardinelli, La poesia verso la prosa. Controversie sulla lirica moderna, Torino, Bollati Boringhieri, 1994, p. 51.
} 
Lo spostamento nel rapporto io-linguaggio-realtà, che parte dalla classica subordinazione del mondo al soggetto che lo guarda, raggiunge il suo culmine proprio negli anni in questione, con la moltiplicazione dell'io e l'idea di una linguacorpo. Tale tendenza coincide con il postmoderno rifiuto delle categorie forti e delle legittimazioni onnicomprensive, che fungeva da base del pensiero debole rappresentato da Gianni Vattimo. ${ }^{13}$ Nelle analisi del filosofo la categoria moderna di Überwindung, cioè di un progressivo superamento dialettico, viene sostituita con quella di Verwindung, apparsa negli scritti tardi di Heidegger. Questa allude, invece, al rimettersi da una malattia, consapevoli di portarne le tracce. È una condizione che porta i segni del passato, cui risalgono le nostre radici, i segni che possiamo provare a distorcere, però mai riusciamo a negare. Le risulta altresì vicino il termine di Andenken, con cui viene descritto lo stato della rimemorazione.

Confrontiamo adesso queste osservazioni con le peculiarità proprie dei testi scritti dagli autori più giovani, antologizzati da Giulia Martini nei volumi Poeti italiani nati negli anni ' 80 e '90. Seguendo le analisi della curatrice, ${ }^{14}$ possiamo ribadire che l'esperienza del corpo rimane strada maestra anche di questo nuovo orizzonte poetico. La disgregazione del mondo si rispecchia qui nelle immagini della malattia ed emana anche sullo spazio circostante, prendendo per esempio forme del cibo nocivo. Accanto a quest'aspetto, imparentato con le dinamiche proprie della categoria di Verwindung, nei testi dei poeti giovani comincia a venire recuperato anche un legame nostalgico con le fondamenta. In uno stato di Andenken poetico, il viaggio di ritorno, il nostos, si sposa con la volontà di partenza verso il futuro, immedesimata nella presenza di mezzi di trasporto, ma anche nelle ricorrenti forme del futuro semplice.

Il ritorno alle radici, alle caverne e una contemporanea nostalgia del futuro non ricreano però il momento poetico che contenga in sé la durata, ma restano ancorati al susseguirsi degli istanti. Ciò avviene perché nel campo poetico il senso della durata non è che la capacità di riconoscersi appieno nel momento presente, mantenendo la coscienza del passato. Le dinamiche, distinte da Martini, non portano a rifondare la continuità tra passato e futuro intrecciati nell'adesso della poesia, ma introducono piuttosto degli idealizzati punti di riferimento: stabiliscono dei ricorsi nostalgici alle fondamenta e provano a proiettarle verso il tempo a venire. Non viene aperto il dialogo, postulato da Vattimo, che radichi un'espressione nel qui-e-ora e a partire dal qui-e-ora costruisca la propria

\footnotetext{
${ }^{13}$ Cfr. Gianni Vattimo, La fine della modernità, Milano, Garzanti, 1985. Dello stesso autore si veda anche Le avventure della differenza, Milano, Garzanti, 1980.

${ }^{14}$ Giulia Martini, Gli oggetti poetici degli anni Venti, Intervento al convegno TwentyTwenty Extended Conference: Interpreting 21st century poetry, 23/02/2021.
} 
dimensione carica degli incontri con il passato, ma si continua invece a sperare una condizione migliore, una liberazione dal fardello del presente. Se questo fosse l'unico modo di affrontare la condizione contemporanea e relazionarsi con il passato e il futuro, sarebbe necessario riassumere tutta la produzione degli ultimi anni con le parole di Remo Ceserani:

Un'altra caratteristica fondamentale della cultura postmoderna è il senso debilitante di un eterno presente, che cancella dall'attenzione del soggetto il passato storico e il futuro, sia nella sua forma utopica sia in quella apocalittica e catastrofica. Ecco che allora nei prodotti culturali di questo periodo si assiste al declino delle tematiche della temporalità, della memoria e della «durata». Il passato diventa un grande serbatoio culturale di immagini, un «immenso simulacro fotografico» da «consumare» con atteggiamento «nostalgico», da Historismus onnivoro e quasi libidico. ${ }^{15}$

Non si cerca qui di negare il valore estetico, ideologico o morale della produzione artistica degli autori più giovani, ma solo evidenziare la paradossalità dell'approccio che bisogna assumere per superare la chiusura della produzione egocentrica. I ricorsi nostalgici al passato, permeati della speranza di costruire un futuro nuovo, non sono che espedienti della compensazione individuale dell'incapacità di affrontare la sottolineata degradazione, la mancanza, l'inefficienza del mondo. Il corpo non viene impegnato come strumento di una cognizione più profonda del reale, ma sembra soprattutto condannato a subire $\mathrm{i}$ dolori del contemporaneo. L'ego spinge in tale contesto a fuggire dal presente, piuttosto che ad accettarlo.

Il tentativo di riprendere i momenti dal passato, come mezzi per redimere il futuro, porta per l'appunto a svuotarli della loro forza vitale. Di conseguenza i ricorsi temporali non possono contribuire alla costruzione del momento presente perché perdono libertà, essendo distorti dai bisogni dell'io empirico. Dopotutto è la nostalgia stessa ad essere espressione del culto di una nostra immagine ideale del presente e non il segno di riconoscimento del passato come tale, perché ciò che è cruciale in questi ritorni non è il passato stesso, ma la nostra idea di quel passato, $\mathrm{i}$ sentimenti che provoca in noi. ${ }^{16} \mathrm{Il}$ continuo riportare delle immagini nell'orizzonte personale, porta alla cancellazione della distanza ed all'impossibilità di revisionare il passato concedendogli l'esistenza autonoma ed oggettiva. Tale fenomeno sembra ripercorrere le dinamiche della perdita dell'aura rilevata da Walter Benjamin. ${ }^{17}$ Bisogna ricordare che nel caso distinto dal critico tedesco: «l'aura dell'opera

\footnotetext{
${ }^{15}$ Remo Ceserani, Raccontare il postmoderno, Torino, Bollati Boringhieri, 1997, p. 88.

${ }^{16}$ Cfr. Marek Zaleski, Formy pamięci, Gdańsk, słowo/obraz terytoria, 2004, p. 18.

${ }^{17}$ Cfr. Walter Benjamin, Di alcuni motivi in Baudelaire, in Angelus Novus. Saggi e frammenti, ed. it. a cura di Renato Solmi, Torino, Einaudi, 1995, pp. 87-160 (ed. or. Frankfurt 1940).
} 
implica una distanza che è da intendere non come una separazione astratta tra l'opera e l'osservatore, bensì come una dimensione che rende impossibile una fruizione immediata dell'opera stessa, obbligando così a una "ad-tensione", e quindi a una comprensione, infinita $\gg .{ }^{18}$ Per intendere meglio il rapporto tra la centralità del corpo e la scomparsa dell'aura al livello da noi analizzato, proviamo a riconsiderare in primo luogo la questione del soggetto poetico, fondando le nostre seguenti analisi sull'esempio dell'ultimo volume di Milo De Angelis.

\section{Il soggetto e il corpo in Linea intera, linea spezzata}

Un confronto con le tendenze che accompagnano il lavoro poetico di Milo De Angelis, e a cui le sue raccolte vengono solitamente attribuite, è necessario per riconoscere propriamente i pilastri fondamentali della sua poetica. Considerando il bilancio fra io e corpo, bisogna affermare che queste due presenze tendono ad intrecciarsi continuamente all'interno dei suoi componimenti. Le lacerazioni che percorrono il discorso lirico di De Angelis vengono rilevate in effetti dagli inizi, fino alla stagione tarda della sua produzione poetica. Roberto Carifi osservò già nel 1982: «In De Angelis l'unità del soggetto deve essere ricostruita altrove, nel luogo metaforico e metastorico del mito, di un'identità primigenia $\gg .{ }^{19}$ Alessandro Baldacci, nel suo recente volume di saggi monografici dedicati all'autore, parla invece di «una soggettività che si colloca al limite fra decentramento dell'io e perentoria affermazione del lirico, fra concentrazione e dilatazione dell'identità (sentita subito da Baudelaire come chiave del lirico moderno), fra prepotente volontà di autoaffermazione e dispendio centrifugo del discorso monologico». ${ }^{20}$ Abbiamo qui a che fare con un approccio in cui non viene cancellato il soggetto, ma non si tenta neanche di reintrodurlo in chiave ermetica. Entrambe le posizioni critiche ci indirizzano verso un'idea dell'io che non scompare del tutto, ma oscilla fra gli opposti, eccede i propri limiti e si scioglie all'interno del linguaggio che crea. Il fulcro di quest'impostazione è il sentimento del limite. Dal dominio del soggetto che coglie il reale tenendosi sempre a distanza, il centro poetico trapassa verso l'esperienza in cui il corpo diventa sia elemento della rappresentazione che la modalità determinante della conoscenza. La presenza del corpo non si esaurisce in un'espressione febbrile ed euforica, retta quasi da un'illusione di aver conquistato la pienezza, la voce non obbedisce alle emozioni che spingono a dimostrare direttamente ciò che percepiscono i sensi, ma il corpo diventa strumento della

\footnotetext{
${ }^{18}$ Giuseppe Di Giacomo, La questione dell'aura tra Benjamin e Adorno, in «Rivista di estetica», 52 (2013), pp. 235-256.

${ }^{19}$ Roberto Carifi, Aspetti teoretico-critici della “nuovapoesia”, in «Nuova Corrente», 89 (1982), pp. 509-534, alla p. 510.

${ }^{20}$ Alessandro Baldacci, Milo De Angelis. Le voragini del lirico, Milano-Udine, Mimesis, 2020, p. 14.
} 
penetrazione dell'orlo dell'impossibile. De Angelis segue a questo punto la linea di Antonin Artaud per cui era proprio «il corpo a consentire l'esperienza del limite (della parola, del contatto fisico, del percepire e del conoscere)», ${ }^{21}$ come osserva giustamente Niva Lorenzini.

Nell'ultima raccolta di Milo De Angelis, Linea intera, linea spezzata, uscita il 26 gennaio 2021 per Mondadori, la soggettività emerge attraverso varie focalizzazioni del discorso poetico. Le rappresentazioni non vengono mai assoggettate ad un io narcisistico che tenda a decidere i limiti del reale. Si rimane lontani dal modello del poeta-vate, con la sua vena gnomica, rivelatrice, estatica, una percezione superiore che conosca il segreto delle cose. Non vi si realizza neanche la poetica dell'eterno istante ontologizzato e astorico. Questa è invece la linea che va da Rimbaud e Campana per arrivare a De Angelis (quel «Rimbaud milanese», ${ }^{22}$ come lo definisce Maurizio Cucchi), essendo la linea dell'io che diventa linguaggio intriso di fisicità. Il corpo è il luogo delle esperienze e il propulsore delle parole, della loro articolazione sull'orlo della dispersione e del silenzio: «il corpo ondeggia ma è deciso a pronunciare | ad alta voce le sue accuse $\gg .{ }^{23}$ Il linguaggio si carica di corporalità sino a diventare cosa tra le cose, dove «l'esordio delle frasi cadde sul pavimento». ${ }^{24}$

Al tempo stesso l'io, mantenendo la sua ascendenza fortemente lirica, resiste in quanto forza capace di far approssimare lo sguardo all'accaduto, permette di scrutarlo da diverse ottiche. Ciò avviene proprio con la sua immedesimazione in varie persone dell'enunciato. Seguendo i testi osserviamo che le due prime sezioni poetiche sono dominate interamente dalla centralità del 'tu'. Il discorso viene rivolto ad un essere vicino che spesso è anche inequivocabilmente riconoscibile, oppure si riveste di un soliloquio quasi come, riconnettendo le epoche, l'io parlasse con se stesso. La voce della prima persona non si limita all'espressione dei sentimenti propri, ma appare attraverso la dimensione condivisa con un interlocutore. Possiamo osservare tali dinamiche nella poesia Piazza Cavalleggeri 2 dedicata a Mario Luzi:

Amico mio, ti vedo, ti vedo in una spiaggia toscana, mentre passa un treno locale con tutti i passeggeri affacciati al finestrino,

\footnotetext{
${ }^{21}$ Lorenzini, Corpo e poesia nel Novecento italiano, p. 7.

${ }_{22}^{22}$ Maurizio Cucchi, Milo De Angelis, il dolore di un poeta, in Cronache di poesia del Novecento, Roma, Gaffi, 2010, p. 447.

${ }^{23}$ Il penultimo discorso di Daniele Zanin, vv. 2-3, in Milo De Angelis, Linea intera, linea spezzata, Milano, Mondadori, 2021 (d'ora in poi LILS), p. 102-104.

${ }^{24}$ Matita blu, v. 10, LILS, p. 15.
} 


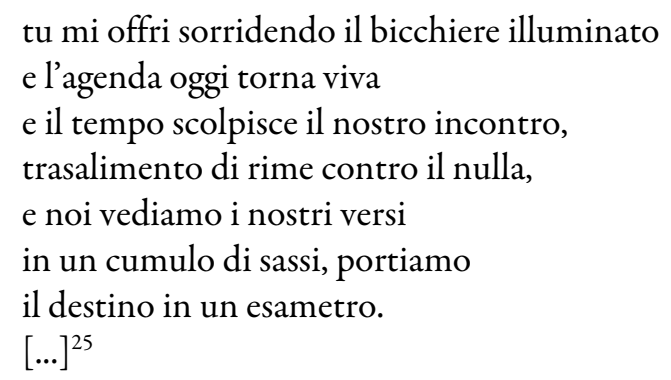

La presenza della seconda persona (solitamente singolare) nei componimenti deangelisiani era presente fin dal suo esordio poetico, come dimostra Marco Villa sull'esempio delle Somiglianze. ${ }^{26}$ Nell'ultima raccolta però il discorso allocutorio diventa costrutto formale che regge interamente le due sezioni, senza permettere spostamenti significativi della voce. Bisogna notare che il rapporto con il 'tu' implica una costante presenza dell'io, contribuendo ad approfondire il carico del lirismo. Se la voce dell'io resta qui ancora prevalentemente nell'ombra, la sua piena messa in scena avviene nella sezione seguente, Dialoghi con le ore contate, dove il soggetto ripercorre i propri tempi, incontrando varie persone, viventi o già scomparse. Le loro parole vengono riportate in quanto discorso diretto, racchiuso nei frammenti virgolettati (Lauretta, A.D.E., Caramelle di menta, Strapiombo e altre ancora), oppure invadono direttamente la dizione dell'io, (si veda Alberico sala). Capita anche che le virgolette abbraccino tutto il componimento, come nel caso di In un bar degli anni Settanta, mettendo l'io nella posizione esterna dell'ascoltatore. L'estensione dei punti di vista raggiunge il proprio vertice nell'ultima sezione. La voce del 'noi' e i versi rivolti al 'tu', vengo intrecciati qui con i vasti monologhi dei suicidi (L'arte di estinguersi, L'ora improtetta, Meteora infuocata nella notte, Peppino), dove diventa sempre più difficile riconoscere a chi appartengano le parole. Assunta questa prospettiva, l'esperienza rappresentata attraverso la prima persona si mostra prima di tutto come esperienza del corpo, il suo avvicinarsi alla realtà: «In questa stanza, in questo disegno abbozzato | a carboncino, colgo l'immagine lunare del tuo volto, | un lampo dentro il sangue, e grido al primo sconosciuto | ho sete ho tanta sete un po' d'acqua». ${ }^{27}$

In Linea intera, linea spezzata possiamo dunque osservare come l'atto di conoscere il mondo, radicato nell'intreccio fra corpo e linguaggio, si espande attraverso il rapporto che l'io lirico (nella sua presenza esplicita o implicita) intrattiene con altre figure: con quelle a cui si rivolge, con cui dialoga oppure ancora

\footnotetext{
${ }^{25}$ Piazza Cavalleggeri 2, vv. 1-11, LILS, pp. 29-30.

${ }^{26}$ Cfr. Marco Villa, La sintassi di Somiglianze. Sulla poesia di Milo De Angelis, Pisa, Pacini, 2019, pp. 161-164.

${ }^{27}$ Ceneri di una visione, vv. 1-4, LILS, p. 87.
} 
con quelle che si impadroniscono del suo discorso. In tal modo si allargano inoltre i limiti del cronotopo. ${ }^{28}$ La reciprocità del rapporto di cui si parla fa sì che $\mathrm{i}$ momenti passati non vengono sottomessi agli scopi del presente, ma lo sguardo lirico, pur accogliendoli al proprio interno, concede loro una certa autonomia. I ricordi non sono mai azionati dalla nostalgia del passato, o più precisamente di noi in quel passato, per riferirsi a questo punto a Jean Starobinski che ricorda come secondo Kant «il nostalgico non desidera tanto il luogo della sua giovinezza quanto la giovinezza stessa». ${ }^{29}$ Le caverne stanno qui dentro ogni cosa e non la precedono. ${ }^{30}$ Le visioni non si volgono neanche a progettare un futuro nuovo perché, come dichiara De Angelis, «Quando avviene l'incontro, non c'è futuro. È un a tu per tu assoluto». ${ }^{31}$ La sua diventa persino una raccolta della memoria che riesce a ricostruire costantemente l'aura degli attimi già stati. De Angelis realizza appieno l'idea emersa nella postfazione al volume di tutte le sue poesie del 2017, dove parla dell' «attimo, che non è mai statico, quando è davvero un attimo destinale. Raccoglie in sé le stagioni, fa convergere in se stesso il tempo che precede e quello che segue». ${ }^{32}$ Il poeta permea il momento presente di ciò che è altro dalla visione immediata del mondo, permette un incontro vero tra lo sguardo e l'oggetto guardato, mentre tutto pare svolgersi in uno stato d'attesa. Al livello del soggetto, il procedimento o scompare del tutto, o è privo di qualsiasi traguardo, diventando un'osservazione del luogo sottratta al tempo. Il punto osservato non è mai indifferente, ma ricambia lo sguardo che gli si concede. Tale approccio corrisponde appunto al concetto di Benjamin, nel cui saggio su Baudelaire leggiamo: $\ll[N]$ ello sguardo è implicita l'attesa di essere ricambiato da ciò a cui si offre. Se questa attesa [...] viene soddisfatta, lo sguardo ottiene, nella sua pienezza, l'esperienza dell'aura $\gg .{ }^{33}$ L'esperienza dell'aura, se trattata come esperienza acquisita attraverso la memoria (seguendo a questo punto le riflessioni di Terry Eagleton), ${ }^{34}$ apre un varco nell'unidimensionalità della poesia, circoscritta alle esperienze intimiste del corpo e del momento presente. Bisogna rilevare che anche le già accennate idee di Gianni Vattimo volgevano al superamento del pensiero debole proprio attraverso

\footnotetext{
${ }^{28}$ Usiamo il termine nel senso datogli da Michail Bachtin, in Estetica e romanzo, Torino, Einaudi, 1979, pp. 231-405.

${ }^{29}$ Cfr. Jean Starobinski, Il concetto di nostalgia, trad. it. Alessandro Serra, in Nostalgia. Storia di un sentimento, a cura di Antonio Prete, Milano, Raffaello Cortina, 1992, p. 100 (ed. or. Paris 1966).

${ }^{30}$ Cfr. R.B., vv. 1-3, in LILS, p. 93: «Vedevi troppo, vedevi ogni dettaglio | della vita e dei suoi sotterranei, vedevi i globuli | sperduti nella caverna, udivi il loro grido».

${ }^{31}$ Milo De Angelis, Colloqui sulla poesia, Milano, La Vita Felice, 2008, p. 141.

${ }^{32}$ Id., Cosa è la poesia?, in Tutte le poesie (1969-2015), Milano, Mondadori, 2017, p. 415.

${ }^{33}$ Benjamin, Angelus Novus. Saggi e frammenti, p. 124.

${ }^{34}$ Cfr. Terry Eagleton, Walter Benjamin or Towards a Revolutionary Criticism, London, Verso, 1981, p. 35.
} 
un rapporto "reciproco" con il passato. L'ontologia nichilista di Vattimo esprimeva un approccio apertosi con la caduta della metafisica, un approccio guidato dall'attenzione all'essere, dalla lettura dei segni e dall'ascolto delle voci che arrivano all'uomo contemporaneo. Il filosofo rivalutava appunto la memoria e il ricordo, il legame con il passato e con la tradizione, richiamandosi in tal modo anche allo spirito oggettivo di Hegel, e facendoci intendere che è proprio il dialogo a costituire la strada maestra della verità.

Lo stesso dialogo poetico è un tratto ricorrente nelle poesie di De Angelis, ${ }^{35}$ legandolo soprattutto al Mario Luzi di Nel magma (1963), ma anche ai «dialoghi non comunicativi» di Vittorio Sereni, i quali, come nota Franco Fortini, «non di rado si vorrebbero istituiti con persone scomparse ». ${ }^{36}$ Proprio questo colloquio con gli attimi trascorsi prende vita in Linea intera, linea spezzata, ricaricandosi ulteriormente delle tonalità blanchotiane di L'attente, l'oubli che De Angelis aveva tradotto all'inizio della sua carriera, nel 1978.

Il dialogo in De Angelis non è soltanto un espediente stilistico, ma una vera e propria riapertura al passato, alla memoria e alla tradizione letteraria. Il nostro autore nella sua idea poetica si volge effettivamente al paradigma romantico, attraversando la linea definita da Guido Mazzoni come classicismo lirico moderno. ${ }^{37}$ Il critico basa le osservazioni sul modello del primo Montale, che va dalla seconda edizione degli Ossi di seppia (1928) fino a La bufera e altro (1956). Si tratta, non a caso, delle raccolte che De Angelis indica come fondamentali per la sua formazione. ${ }^{38}$ L'approccio distinto procede per un rapporto dialettico e, attingendo al grande modello romantico europeo della poesia soggettiva di tono tragico, tiene conto dei cambiamenti moderni, senza abbandonarsi alla nostalgia del passato o ai toni ironici che esaltino la stessa frattura avvenuta nel secondo Novecento. Tale disposizione viene incorporata nel linguaggio preciso, attento alle sfumature dei sensi, volto ad esprimere con nitidezza le esperienze particolari. Ciò che differenzia questa linea dal modello canonico è la discontinuità dell'andamento dell'espressione poetica, il che significa che lo sviluppo temporale procede per rivelazioni ed epifanie piuttosto che per un'idealizzazione totale del quadro lirico. «Se le biografie romantiche sono potenzialmente continue», afferma Mazzoni, «giacché in ogni istante la vita personale può trasformarsi in un simbolo universalmente umano, gli attimi densi e pieni cui le poesie montaliane danno voce

\footnotetext{
${ }^{35}$ Cfr. Laura Toppan, De Angelis e Luzi: dialogicità e interrogazione in poesia, in L'avventura della permanenza. La poesia di Milo De Angelis, a cura di Alberto Russo Previtali e Jean Nimis, MilanoUdine, Mimesis, 2020, pp. 147-164.

${ }^{36}$ Franco Fortini, I poeti del Novecento, Roma, Donzelli, 2017, p. 184.

${ }^{37}$ Cfr. Mazzoni, Sulla poesia moderna, pp. 187-189.

${ }^{38}$ Cfr. De Angelis, Colloqui sulla poesia, p. 107.
} 
sono del tutto occasionali, essendo circondati da un tempo vuoto e insensato $\gg .{ }^{39}$ De Angelis chiaramente non riprende il legame formale (metrico, sintattico, lessicale) con la lirica premoderna, come succede nel caso di Montale; tuttavia, il cardine del classicismo lirico moderno non è la rigidità stilistica, ma la medesima capacità di instaurare una continuità dialettica e vitale tra la linea romantica e le dinamiche dei tempi nuovi. Nell'ultimo volume di De Angelis è proprio questa la tendenza che si fa sentire decisamente di più rispetto al mito delle origini assegnato giustamente da Gianluigi Simonetti alla prima fase della sua creazione poetica, quando dominava ancora «l'emozione di una storia che inizia», piuttosto che ricorsi al passato. ${ }^{40}$

\section{L'importanza dello spazio. Il modello della generazione di mezzo}

L'intrecciarsi delle voci nell'opera di De Angelis, svincolato dall'idea della storicizzazione progressiva, si fonda su una cartografia precisa di Milano: «non corrisponde a un'assenza di radici, sostando anzi in un hic et nunc particolarmente solido», ${ }^{41}$ ribadisce Giorgia Bongiorno. Il rapporto con lo spazio, ereditato in gran parte dai poeti della generazione di mezzo, sembra costituire persino un ordito dei suoi incontri lirici, ma anche un discrimine rispetto alle poesie circoscritte esclusivamente alla percezione sensibile della materia e alla disgregazione contemporanea del mondo. Milano costituisce qui un tessuto che «si mescola fitto alle parole, si impasta con esse $\gg .{ }^{42}$ Il linguaggio si riempie con la presenza fisica delle strade lombarde, mentre i luoghi assumono dei tratti umani. Citiamo De Angelis: «Avviene che i luoghi ci chiamano, si rivolgono a noi e noi andiamo lì dove siamo stati chiamati, chiamati a giudizio naturalmente, e succede che allora, nella commozione assoluta del corpo, ogni dettaglio - i muri di una casa o una curva di una strada o una tangenziale che si profila - diventa decisivo». ${ }^{43} \mathrm{La}$ 'commozione del corpo' è una commozione reciproca che apparenta l'io con la città, inserisce l'individuo nell'orizzonte ed attribuisce la percezione umana allo spazio circostante. I luoghi, sempre presenti nella scena complessiva della sua opera, ${ }^{44}$

\footnotetext{
${ }^{39}$ Mazzoni, Sulla poesia moderna, p. 188.

${ }^{40}$ Cfr. Simonetti, La letteratura circostante. pp. 164-167.

${ }^{41}$ Giorgia Bongiorno, La dinamica dell'istante urbano nella poesia di Milo De Angelis, in L'avventura della permanenza. La poesia di Milo De Angelis, p. 59.

${ }^{42}$ Ibid.

${ }^{43}$ Milo De Angelis, in La poesia a scuola. A colloquio con i poeti, a cura di Carlangelo Mauro, Enzo Rega, Roma, Antonio Stango Editore, 2003, p. 34.

${ }^{44}$ Della presenza di Milano, da quella "esistenziale" delle Somiglianze a quella "metafisica" del Distane un padre» tratta Erardo Affinati in Patto giurato. La poesia di Milo De Angelis, Pescara, Tracce, 1996. A questo proposito si veda ancora lo stesso De Angelis, Colloqui sulla poesia, p. 168:
} 
anche nell'ultima raccolta azionano tutti i ricorsi al passato: «Dovevo ritornare, lo so, ma non per te, mia invincibile amica, | e nemmeno per le vostre voci ritrovate... dovevo tornare | per un oscuro richiamo dei luoghi», ${ }^{45}$ leggiamo in Piscina Scarioni.

A proposito del nostro richiamo ai poeti della terza generazione, dobbiamo aprire una breve parentesi e riferirci alle osservazioni fatte in merito da Roberto Galaverni nel libro Dopo la poesia. Saggi sui contemporanei. Il critico fonda le sue ricerche proprio sul senso del luogo che indirizza lo sguardo poetico verso «qualcosa di più ampio e di eccedente», «qualcosa che sta dietro, che viene dal fondo: una musica stranamente lontana, arcana ma famigliare». ${ }^{46}$ Tale prospettiva porta ad esaltare non solo la costante presenza dei richiami topografici, ma evidenzia l'aspetto della memoria dei luoghi inclusa nelle opere in questione. Le analisi di Galaverni partono dal modello di Seamus Heaney e la sua nozione del luogo fondata sull'estensione analogica. Essa non procede per idealizzazione o abbellimento dello spazio perduto, ma recupera un aggancio vitale tra il momento presente e le sue origini, «come se egli volesse discendere, attraverso il proprio luogo, giù giù fino al centro del mondo». ${ }^{47}$

Pare essere proprio il distacco spazio-temporale, introdotto dalla memoria, a permettere questa riscoperta, come se una vicinanza eccessiva togliesse allo sguardo le stesse possibilità cognitive, limitando la percezione all'empirismo puro. Il ritorno diventa un recupero del sé, della propria identità rimasta legata ad un orizzonte da tempo scomparso. Non si cade però nel sentimentalismo, nell'elogio soggettivista di una patria edenica che attraversi una presunta lingua dell'autenticità. L'immagine viene forgiata nel linguaggio intriso di concretezza, denudato ma vitale, lontano dai toni nostalgici e capace di saldare il reale con l'eterno. La sovrapposizione fra due momenti non provoca una rarefazione linguistica della realtà esterna, ma al contrario accentua ulteriormente l'esistenza sensibile degli oggetti. I testi non relegano visioni poetiche in uno spazio ideale, ma rendono presente la mancanza stessa.

Lo spazio diventa in questo modo il mezzo per aprire un varco nell'impossibilità di ristabilire il legame fra storia e assoluto, sottolineata da Berardinelli. La nozione del luogo permette ai poeti della generazione di mezzo di rivendicare un rapporto dinamico fra la percezione sensibile del reale e un'esperienza vera di ciò che lo eccede. «I luoghi», così Galaverni, «assottigliano la loro consistenza concreta,

\footnotetext{
«Il paesaggio è davvero una necessità primordiale. Non riesco a ricordare un solo incontro della mia vita fuori da un paesaggio urbano che rimane sempre lo stesso».

${ }^{45}$ Piscina Scarioni, vv. 9-11, LILS, p. 84-85.

${ }^{46}$ Roberto Galaverni, Dopo la poesia. Saggi sui contemporanei, Roma, Fazi Editore, 2002, pp. 12-13.

${ }^{47}$ Ivi, p. 13.
} 
storico-geografica, e assumono uno statuto contraddittorio, diventando ambivalenti, depistanti, allusivi, indecidibili, come di un qui a cui si sovrappongono o su cui si proiettano altri tempi e altri territori, altre luci e altri orientamenti ${ }^{48}$

A queste dinamiche sembra corrispondere l'immaginario deangelisiano dove lo spazio centrale resta costantemente quello delle borgate milanesi che una volta concretizzate in Comasia o Bovisa, si sciolgono un attimo dopo nell'immagine di un oceano minaccioso che circondi il capoluogo lombardo. ${ }^{49}$ Per De Angelis, Milano: «più che una città riconoscibile, è un luogo di tensioni, silenzi, analogie $\gg .{ }^{50}$ Essendo una costante della sua poetica, lo spazio dai confini milanesi diventa quel «luogo metaforico e metastorico», postulato da Carifi, il luogo dove viene ricostruita l'unità del soggetto. Tale approccio anima anche le visioni dell'ultimo volume, dove la città diventa un punto calamita e, come abbiamo già sottolineato, non si avvia qui un viaggio nostalgico di ricerca delle fondamenta, né progetto per i tempi a venire; «Il prima e il dopo convergono in sasso», il mondo scompare «dentro un colpo senza origine» e la voce incarna «l'estinzione del futuro». ${ }^{51}$

Il basarsi dell'idea poetica sul senso del luogo viene in fondo legato alla rivendicazione di un'esistenza organica e compiuta dell'io, ossia alla «priorità della coscienza e della libertà individuale»..$^{52} \mathrm{La}$ formazione dei poeti della terza generazione risale in effetti al momento in cui lo statuto assoluto e prioritario del soggetto lirico era ancora innegabile. L'apertura del genere ai nuovi territori, avvenuta nel secondo dopoguerra, restava bilanciata dalla proiezione della misura interiore sullo spazio esterno. Di conseguenza questa nuova inclusività poetica era pur sempre lontana dalla mimesi diretta del mondo, esaminandolo secondo i principi di uno sguardo individuale. Gli ideali assoluti della forma ermetica non vengono qui completamente aboliti, ma trapassano nell'intensità e nell'altezza lirica innestate adesso all'interno di una prospettiva decisamente orizzontale. I versi si aprono al mondo, seguono la molteplicità del divenire, permettono incontri sempre collocabili in un cronotopo, infrangendo in questo modo il modello astrattivo.

\footnotetext{
${ }^{48}$ Ivi, p. 22.

${ }^{49}$ Cfr. L'oceano intorno a Milano che è il titolo della prima sezione di Biografia sommaria. Inoltre, in Colloqui sulla poesia, p. 87, il poeta parlando di Milano afferma: «È una città di naufragi e naufraghi, e mi piace immaginarla circondata da un oceano minaccioso», mentre in Doppio passo di Linea intera, linea spezzata ritorna la figura de «l'oceano dell'infanzia».

${ }^{50}$ De Angelis, Colloqui sulla poesia, p. 73.

${ }^{51}$ Penultima tappa del viaggio notturno, v. 4, LILS, pp. 40-41; Per l'Adele, v. 19, ivi, pp. 25-26; 21 settembre, v. 6, ivi, p. 20.

${ }^{52}$ Galaverni, Dopo la poesia. Saggi sui contemporanei, p. 25.
} 
Si può osservare, soprattutto nella stagione tarda di questi poeti, una sorta di divaricazione delle tensioni poetiche: un costante aggancio al piano storicoesistenziale e un contemporaneo interesse verticale. Il soggetto non riesce a riconoscersi appieno nel mondo, non può limitarsi alle sue strade. L'estraneità personale e una visibile inclinazione verso un altrove portano conseguentemente alle modificazioni sensibili dello spazio, però mai al suo definitivo abbandono. La voce poetica continua a sperimentare i limiti del mondo, la sua finitudine, il che si evidenzia anche nel linguaggio rimasto proprio della descrizione orizzontale del presente. La dimensione offerta dal luogo concreto, sebbene insufficiente, continua a mostrarsi però come ambito indispensabile per la autocomprensione dell'io e la creazione del testo poetico. L'opera non è subordinata allora allo status del mondo esterno, non è spinta a disfarsi in seguito alla sua disgregazione, ma viene retta dalla centralità del soggetto parlante che costruisce la propria identità in rapporto ai luoghi costitutivi per il suo essere e quindi, come nota Galaverni, «l'oscurarsi degli scenari esterni entra sempre in contrasto, come per uno strano effetto di rifazione, con l'inesausta ricchezza propositiva della dimensione interiore $\gg .{ }^{53}$

Il contesto della generazione di mezzo ci è servito ad evidenziare come la presenza dello spazio possa diventare un'emanazione del soggetto parlante. Ed è proprio la poetica di De Angelis a fondarsi su un percorso esperienziale radicato nella nozione del luogo. Il suo allontanamento dalla purezza formale porta all'estensione degli orizzonti del lirico. Tuttavia, vi viene pur sempre mantenuta un'ottica fortemente soggettiva: la visione del mondo trapassa la figura dell'io ed anche quando viene concessa libertà agli oggetti, l'io sembra sciogliersi al loro interno. La poesia recupera la corporeità, rimossa nella poesia ermetica, senza ridursi al dominio dell'esperienza narcisistica del corpo. Lo spazio diventa così anche il luogo della memoria, essendo uno stabile punto di riferimento senza il quale le visioni non potrebbero mai manifestarsi. Conseguentemente i ricordi che nascono dallo spazio permettono anche un'estensione temporale ed introducono la dimensione della durata.

\section{Soggetto, spazio e memoria nelle ultime poesie di De Angelis}

Le poesie di Linea intera, linea spezzata mantengono una costanza, persino sorprendente, del percorso se le confrontiamo con le opere della generazione più giovane, ma anche con i componimenti precedenti di De Angelis. La voce poetica, esistente fuori dal tempo però mai fuori dallo spazio, procede piuttosto per metamorfosi che per immagini slegate, conducendo il lettore a ritrovare i momenti già stati, le persone, le date. E così vediamo in questi versi un incontro poetico con

\footnotetext{
${ }^{53}$ Ivi, p. 53.
} 
Piero Bigongiari (Piazza Cavalleggeri 2), Gottfried Benn (Quinta tappa del viaggio notturno), con i versi di Montale (Libertà vigilata), ma anche con Cesare Pavese (Mercoledi). Lo sguardo ripercorre le scene avvenute al liceo (Scrutinio finale), all'università (Dialogo con un compagno), attraversa la prima visione de Il grido di Antonioni (Un film chiamato "Il grido"), arrivando anche ai periodi irraggiungibili con la sola memoria personale, come Belle Epoque. In questa raccolta lo sguardo lirico sembra disegnare un percorso che abbraccia il tempo nel suo intero, il che viene espresso anche attraverso gli intrecci grammaticali fra indicativo presente e passato remoto: «senti lo stesso odore di cloroformio | dove affondò la veste di lino» oppure fra passato prossimo e futuro semplice: «questo è stato il mio tempo, | un tempo di dischetti e figurine, e qui resterò per sempre». ${ }^{54}$ Più si va avanti attraverso le visioni, più rallenta il ritmo del susseguirsi delle scene reali. Le parole non rispecchiano nessun progresso, avendo perso del tutto il loro aggancio al tempo della storia. Si è invasi da un sentimento del percorso impercorribile che al contempo si mostra come costante discesa e riaffermazione del già stato. Tali dinamiche appaiono subito nella poesia d'apertura del volume, intitolata Nemini:
Sali sul tram numero quattordici e sei destinato a scendere in un tempo che hai misurato mille volte ma non conosci veramente, osservi in alto lo scorrere dei fili e in basso l'asfalto bagnato, l'asfalto che riceve la pioggia e chiama dal profondo, ci raccoglie in un respiro che non è di questa terra, e tu allora guardi l'orologio, saluti il guidatore. Tutto è come sempre ma non è di questa terra e con il palmo della mano pulisci il vetro dal vapore, scruti gli spettri che corrono sulle rotaie e quando sorridi a lei vestita di amaranto che scende in fretta i due scalini, fai con la mano un gesto che sembrava un saluto ma è un addio. ${ }^{55}$

Il soggetto sembra essere condotto in un altro tempo, guardandolo però solo dall'esterno; non può intervenire nel succedersi delle cose, non può modificare il loro ordine. Il dialogo interno di questo componimento parte con la salita sul tram; tuttavia, la presenza di un mezzo di trasporto (analogamente succede in Settima tappa del viaggio notturno, Lauretta e Alberico sala) non testimonia la propensione verso il mondo, il tentativo di avvicinarsi ad un traguardo e costruire un nuovo futuro: le dinamiche distinte invece da Martini nei versi dei poeti nati nell'ultimo

\footnotetext{
${ }^{54}$ Penultima tappa del viaggio notturno, vv. 14-15, LILS, pp. 40-41; A.D.E., vv. 21-22, ivi, pp. 4748.

${ }^{55}$ Nemini, LILS, p. 9.
} 
ventennio del Novecento. In De Angelis la partenza rievoca un viaggio memoriale che, come l'aveva definito una volta l'autore stesso, segue la linea spirale, senza coincidere mai pienamente con il momento passato. ${ }^{56} \mathrm{Il}$ poeta introduce in tal modo la prospettiva dell'esilio che si mostra adesso come un esilio consapevole o già provato, lontano dalla disperazione che l'aveva accompagnato nelle raccolte degli anni Ottanta. Inoltre, le figure che riemergono dal tempo sono meno scarne, sembrano essere strettamente legate agli attributi della metropoli lombarda, alla sua topografia esatta, avvolte nell'asfalto, nella pioggia, nel vapore sui vetri, nella nebbia, ma anche in colori che illuminano le scene, in profumi di vaniglia, verbene (Per l'Adele), viole (Quinta tappa del viaggio notturno), oleandri (Libertá vigilata), dalie (Comunitá incontro), fino ad ora mai presenti. Di sottofondo riemerge la musica in cui si radunano le voci del passato. Essa è soprattutto presente nella sezione Nove tappe del viaggio notturno:

Più tardi, nel silenzio della via, hai trovato una lampada hai intonato la dolce canzone degli astri in movimento, sei arrivato di fronte all'antico portone.

Ricordiamo, ricordiamo esattamente. [...]

Hai camminato e sei giunto in una grande sala dove uomini vestiti di scuro ascoltano la cantante dall'abito turchese e percorrono un cammino che va dall'inizio più remoto a questo pianoforte ascoltano la sua voce sempre più roca dove mille voci che credevi disperse ritornano vive [...]

$[\ldots]$ intoni l'ultima nota di un'immensa sinfonia, con la voce di tutti gli affogati. ${ }^{57}$

Tutto ciò sembra ricreare l'aspetto auratico dei punti passati, il quale aziona il flusso della memoria poetica. Se gli incontri con le persone scomparse erano già presenti in De Angelis, soprattutto nelle poesie di Quell'andarsene nel buio dei cortili e Incontri e agguati, rimanevano abbozzati dalle intermittenze dello sguardo,

\footnotetext{
${ }^{56} \mathrm{Cfr}$. De Angelis, Tutte le poesie, pp. 414-415: «un tempo che, ritornando, si avvicina alla strada dell'andata e può dunque osservarla e percepirla senza coincidere con essa. La figura geometrica che disegna questa forma non è dunque il cerchio, ma la spirale»; a questo proposito si veda anche Milo De Angelis, "L'interminabile parola data", lettere a Damiano Sinfonico, in La parola data (interviste 2008-2016), Milano-Udine, Mimesis, 2017, p. 56.

${ }^{57}$ Terza tappa del viaggio notturno, vv. 1-4, LILS, p. 35; Quinta tappa del viaggio notturno, vv. 1-6, ivi, p. 37; Penultima tappa del viaggio notturno, vv. 16-17, ivi, pp. 40-41.
} 
senza creare una visione così definitiva. In Linea intera, linea spezzata si percepisce un cambio di tonalità e di temperature di questi attimi. De Angelis riesce a permeare le parole del furore immerso nella quiete, ricollega l'elemento dionisiaco con quello apollineo, esprimendo con questo strumento l'atmosfera del momento in cui la vicenda è già passata, lo stadio è già chiuso, una volta finita la gara, ma la polvere agitata dagli atleti non è ancora caduta per terra, come se i veri avvenimenti non avessero contenuto tutta la forza che li aveva messi in movimento e continuano a starvi ancora. Tale momento, amato dal nostro autore fin dalle poesie d'esordio, ${ }^{58}$ dona l'intensità ai versi della sua ultima raccolta, dove gli incontri vengono in qualche modo protratti, prolungati in un'immagine più complessa. L'autore ritorna ai momenti passati, ma anche riaggancia il momento stesso di tale ritorno ad un momento analogo e già avvenuto: riprende le proprie parole saldando una sorta di dimensione rituale della poesia. ${ }^{59} \mathrm{E}$ così, nel verso «Ricordiamo, ricordiamo esattamente» della Terza tappa del viaggio notturno risuonano - come nota Francesco Perardi ${ }^{60}$ - le parole della Cartina muta della Biografia sommaria: «Riportiamo esattamente | i fatti e le parole». ${ }^{61} \mathrm{Si}$ fondano in tal modo delle immagini a doppio specchio, dove in forza delle stagioni passate - ed attingendo ancora di più al pensiero di Pavese, alla nozione della capacità conoscitiva del secondo sguardo - nasce l'imperativo: «adesso devi restare, devi restare, devi dare alla notte | la sua dizione più precisa $\gg .{ }^{62}$ Si percepisce qui una maggiore capacità di avvicinarsi al passato che permette di rendere più netti i particolari. I ritorni prendono forma nella sovrapposizione dei piani temporali il cui fondamento resta sempre quello di un luogo concreto. In tal modo le poesie deangelisiane continuano a costruire un repertorio della memoria dei luoghi.

Un po' come nel caso di Vittorio Sereni, neanche in De Angelis Milano è soltanto la Milano reale, il qui non è solo qui, l'adesso non è solo adesso. Nei versi del poeta luinese i luoghi, pur conservando «il valore delle cose irripetibili», «sono aperti per eccellenza, costruiti come per una fluida intersezione di piani moltiplicati $\gg ;{ }^{63}$ fondati su una solidità memorabile, stanno in un dinamico equilibrio fra vitalità e morte. Attraverso l'apertura allo spazio esterno si esprime un attrito tra io e mondo. La poesia entrando nel dialogo con le cose continua a

\footnotetext{
${ }^{58}$ Cfr. De Angelis, Colloqui sulla poesia, p. 162.

${ }^{59}$ Per approfondire l'aspetto dei ritorni e delle autocitazioni in De Angelis si veda soprattutto Andrea Afribo, Deangelisiana, in Poesia italiana postrema. Dal 1970 a oggi, Roma, Carocci Editore, 2017, pp. 107-126.

${ }^{60}$ Francesco Perardi, La forza centripeta conduce l'universo, in «L'Indice dei libri del mese», 6 (2021), p. 38

${ }^{61}$ De Angelis, Tutte le poesie, p. 265.

${ }^{62}$ Bowling dei fiori, vv. 10-11, LILS, p. 14.

${ }^{63}$ Galaverni, Dopo la poesia. Saggi sui contemporanei, p. 33.
} 
salvaguardare la soggettività dello sguardo. Il qui non viene ridotto alla rappresentazione effettiva dello spazio reale, essendo prima di tutto la sua base innegabile, quasi un ordito fenomenologico del mondo sereniano. Tale impostazione si evidenzia soprattutto nella sua ultima raccolta Stella variabile (1981) che, come afferma Galaverni, è «una raccolta non localizzabile in modo univoco, ma decentrata, topograficamente eccentrica ed eccedente, variabile, proprio come indicato dal titolo»..$^{64}$ Per aprire i luoghi ad un significato vivo e insopprimibile, indipendente dall'esaurimento della dimensione storicoesistenziale, Sereni sa anche liberare lo spazio dai personaggi concreti, universalizzando le loro presenze. De Angelis invece non tende mai a rendere assoluti gli elementi delle sue immagini, ma continua ad entrare in un contatto vero con il passato. Un'attenta percezione dei luoghi permette lui di accogliere i momenti già vissuti, «anni che sono rimasti | fuori dalla morte». ${ }^{65}$ Seguendo questa linea, ritroviamo in De Angelis il nodo di quel rapporto reciproco tra soggetto, spazio e memoria. L'ottica individuale non distorce il ricordo perché è lo spazio a farlo riemergere ed a garantire la sua autonomia, mentre il soggetto si manifesta soprattutto in forza dello sguardo concesso allo spazio.

Come si cerca di sottolineare, la sua memoria poetica non è volta a salvare i ricordi, creando un idilliaco spazio poetico che diventi un rifugio dallo scorrere del tempo, ma apre la dimensione dell'incontro dove tutti gli attimi recuperano la propria forza e dove anche il momento della morte viene intensificato fino ad eccedere il proprio limite. In riferimento al penultimo volume di De Angelis, Incontri e agguati, Luigi Tassoni afferma che è proprio la «voce nel testo [...] a raccontare come testimone, guida e ascolto, la non istantaneità della morte, il suo intrecciarsi all'esistenza, giacché per il poeta il senso della fine e del niente non è azzerante e ultimativo, e il morire è leopardianamente fatto da istanti che consentono di ridisegnare il profilo della vita stessa». ${ }^{66}$ Ciò succede anche nell'ultima raccolta dove la moltiplicazione delle voci ritaglia diverse ottiche sul momento della fine. L'ultima sezione del volume, Aurora con rasoio (inaugurata con una citazione di Arrigo Boito: «... corre levando impetuosi gridi | una pallida giostra | di poeti suicidi»), è proprio una sezione che sembra essere esplicitamente una sezione di morte, ma dopo, più che di morte risulta essere una sezione dell'aumento d'intensità, dell'accumulo delle circostanze che accompagnano la fine. Non si riesce però ad identificare qui il punto preciso della morte né riconoscere con certezza il soggetto che la fa avvenire. In un momento essa sembra

\footnotetext{
${ }^{64}$ Ivi, p. 59.

${ }^{65}$ Per l'Adele, vv. 16-17, LILS, pp. 25-26.

${ }^{66}$ Luigi Tassoni, Il gioco infinito della poesia. La lettura dei componimenti da Ungaretti a De Angelis, Roma, Giulio Perrone Editore, 2021, pp. 210-211.
} 
imminente e sempre più vicina, per collocarsi un attimo dopo già alle nostre spalle. In entrambi i contesti la fine viene espressa soprattutto con le forme verbali del futuro semplice:

Lo farò un giorno di pioggia, lo farò all'aperto, non voglio sporcare la stanza, lo farò di notte nel Ticino.

[...] cancellerò

ogni traccia sul computer, getterò il cellulare, getterò

tutti i quaderni, fogli, agende, tutto finirà nel nulla [...].

Nel buio di un mattino te ne andrai anche tu e scorderai le tue mani le tue frasi le tue estati di poesia $[\ldots] .^{67}$

La morte non è il punto finale del cammino, ma una condizione che continuerà a dilagare, a percorrere il mondo che resta. Essa pare vicina alle dinamiche de L'attesa, l'oblio di Blanchot, dove il protagonista: «cercava di dire tutto perché tutto fosse dimenticato, confidando ciò che è immortale a un individuo passeggero. Dimenticava. Ed era, in un certo senso, l'oblio e la bellezza visibile di ciò che fu dimenticato». ${ }^{68}$ Morire per il mondo prende a questo punto il significato di diventare un ricordo dimenticato. Lo scopo dell'espressione testuale consiste qui nel continuo lavoro, nell'abitare il nulla e il silenzio, che si sottrae al tempo presente, dove l'eternità entra nei momenti consueti e passeggeri, li lega attraverso la memoria, sempre libera dai traguardi. Per De Angelis l'esperienza del ritorno memoriale è in fondo un'esperienza della morte, di scomparire per il mondo, di entrare nell'oblio. Tale aggancio fra memoria e morte era già presente nella poetica di De Angelis, che in una delle interviste affermò: «Il luogo intero è quando all'improvviso ritorna ciò che abbiamo amato, quando i vivi e le ombre si parlano con sapienza [...]. Il luogo intero deve essere da quelle parti, deve essere la morte». ${ }^{69}$ I versi dell'ultima sezione della raccolta, sempre lontani dal tono luttuoso, si fondano inoltre sulle frequenti immagini ossimoriche che esaltano ulteriormente la vita che continua oltre l'atto definitivo della morte: incontriamo qui il buio percorso dal fuoco (Meteora infuocata nella notte), «le bacche gelate dall'inverno» (L'ora improtetta). Si sente costantemente qualcosa che sta per esplodere da dentro, dal sangue che percorre le cose. Sono anche i profumi a diventare più forti, più aspri, dal limone all'aceto sino al catrame (Brown sugar), mentre al posto del canto

${ }^{67}$ L'arte di estinguersi, vv. 1-2, 6-8, LILS, p. 77; Filastrocca del nome perduto, vv. 1-3, ivi, p. 100.

${ }^{68}$ Maurice Blanchot, L'attesa, l'oblio, trad. di Milo De Angelis, Milano, Guanda, 1978, p. 57 (ed. or. Paris 1962).

${ }^{69}$ De Angelis, Colloqui sulla poesia, p. 87. 
appare «Un boato nelle orecchie, un cigolio delle cicale morenti, | un grido impazzito di grilli». ${ }^{70}$ Persino lo stesso titolo della sezione, Aurora con rasoio, sembra indicare l'aspetto postumo di questa visione poetica. Il mattino è in effetti il punto dominato dalla presenza del nulla, il momento che arriva dopo la fine.

\section{Conclusioni}

Riassumendo le analisi, possiamo dire che Milo De Angelis nelle sue ultime poesie non limita la dizione poetica ad una rappresentazione narcisistica dell'esperienza corporale, ma concede ai versi un ordine a parte che, liberatosi dal procedere del tempo, si costruisce a partire dalla nozione dello spazio. I componimenti, percorsi dai flussi di memoria, permettono dei veri e propri incontri con i momenti passati, senza cedere mai a toni nostalgici, al ripiangere ciò che ci è stato tolto, se non «quel battere tumultuoso del sangue $\gg .^{71} \mathrm{Vi}$ avviene un recupero delle forze vitali (o persino dionisiache) che illumina il presente. Il riconoscimento in questo quadro della centralità della voce lirica non deve significare allora un ritorno al modello ermetico. De Angelis percepisce ed entra nel paradosso che si insedia alle basi della creazione poetica: non sovraccaricando l'opera con la sola esperienza del corpo, può mantenere una netta posizione centrale del soggetto poetico. Rivendicando i diritti della voce stessa, non della persona reale, l'espressione poetica permette un'esperienza profonda della realtà del segno. Nei componimenti vengono recuperate le coordinate oggettive della produzione testuale che non viene mai trattata come materiale indistinto, adoperato per dar spazio alle idiosincrasie personali. In questo senso De Angelis pare approssimarsi alla figura del poeta-testo ideata da Zanzotto, una figura del poeta che privilegiando la parola poetica, non si esaurisce «nella matericità fonetica e visiva della performance $\gg .{ }^{72} \mathrm{Di}$ conseguenza, recuperata l'autonomia dello spazio poetico, scompare anche la necessità di imporre alle parole l'obbligo della rivelazione di una verità suprema:

[...] Pregavi.

Ma non per risorgere o per un altro

sogno celeste. Chiedevi un'altra giornata.

Chiedevi di non compiere adesso

il volo deciso dai lampi,

chiedevi di illuminare l'ora solitaria,

chiedevi un'arte più serena di te,

un tenero negozio umano

\footnotetext{
${ }^{70}$ Strada statale 31, vv. 1-2, LILS, p. 91.

${ }^{71}$ L'ora improtetta, v. 7, LILS, p. 80.

${ }^{72}$ Lorenzini, Colangelo, Poesia e storia, p. 270.
} 
dove troverai le stagioni

perdute che rinascono stasera. ${ }^{73}$

Attraverso gli incontri con i momenti recuperati dalla memoria, il soggetto poetico sembra retrocedere, cancellando le tracce della sua esistenza. Ma il suo procedere all'indietro nel tempo fa in realtà ritornare tutti gli attimi già stati, facendo rivivere il passato nello spazio della poesia. Moltiplicando le voci, distende persino il momento della morte e rende più complessa la percezione dell'esistere, per recuperare infine, senza toni lamentosi né profetici, quel silenzio virile che orientava sempre il suo percorso: «la mia | sbilenca estrema azione per salvarmi dal paradiso celeste | o socialista, per tornare finalmente tra gli eroi, | tra i nostri eroi, per tornare nell'immenso nulla valoroso, | e solitario». ${ }^{74}$

\section{Bibliografia}

Erardo Affinati, Patto giurato. La poesia di Milo De Angelis, Pescara, Tracce, 1996.

Andrea Afribo, Poesia italiana postrema. Dal 1970 a oggi, Roma, Carocci Editore, 2017.

Michail Bachtin, Estetica e romanzo, Torino, Einaudi, 1979.

Alessandro Baldacci, Milo De Angelis. Le voragini del lirico, Milano-Udine, Mimesis, 2020.

Walter Benjamin, Di alcuni motivi in Baudelaire, in Angelus Novus. Saggi e frammenti, ed. it. a cura di Renato Solmi, Torino, Einaudi, 1995, pp. 87-160 (ed. or. Frankfurt 1940).

Alfonso Berardinelli, La poesia verso la prosa. Controversie sulla lirica moderna, Torino, Bollati Boringhieri, 1994.

Maurice Blanchot, L'attesa, l'oblio, Milano, Guanda, 1978 (ed. or. Paris 1962).

Simone Carella, Paola Febbraro, Simona Barberini (a cura di), Il romanzo di Castel Porziano: tre giorni di pace, amore e poesia, Viterbo, Stampa alternativa, 2015.

Roberto Carifi, Aspetti teoretico-critici della "nuova poesia", in «Nuova Corrente», 89 (1982), pp. 509-534.

${ }^{73}$ Stille Nacht, vv. 5-14, LILS, pp. 18-19.

${ }^{74}$ In un bar degli anni Settanta, vv. 9-13, LILS, pp. 62-63. 
Remo Ceserani, Raccontare il postmoderno, Torino, Bollati Boringhieri, 1997.

Maurizio Cucchi, Cronache di poesia del Novecento, Roma, Gaffi, 2010

Milo De Angelis, Colloqui sulla poesia, Milano, La Vita Felice, 2008.

Milo De Angelis, Linea intera, linea spezzata, Milano, Mondadori, 2021.

Milo De Angelis, La parola data. Interviste 2008-2016, Milano-Udine, Mimesis, 2017.

Milo De Angelis, Tutte le poesie (1969-2015), Milano, Mondadori, 2017.

Giuseppe Di Giacomo, La questione dell'aura tra Benjamin e Adorno, in «Rivista di estetica», 52 (2013), pp. 235-256.

<http://journals.openedition.org/estetica/1626>

Terry Eagleton, Walter Benjamin or Towards a Revolutionary Criticism, London, Verso, 1981.

Franco Fortini, I poeti del Novecento, Roma, Donzelli, 2017.

Damiano Frasca, Caroline Lüderssen, Christine Ott (a cura di), Costruzioni e decostruzioni dell'io lirico nella poesia italiana da Soffici a Sanguineti, Firenze, Cesati, 2015.

Roberto Galaverni, Dopo la poesia. Saggi sui contemporanei, Roma, Fazi Editore, 2002.

Niva Lorenzini, Corpo e poesia nel Novecento italiano, Milano, Bruno Mondadori, 2009.

Niva Lorenzini, Il presente della poesia, Bologna, il Mulino, 1991.

Niva Lorenzini, Stefano Colangelo, Poesia e Storia, Milano, Bruno Mondadori, 2013.

Carlangelo Mauro, Enzo Rega (a cura di), La poesia a scuola. A colloquio con i poeti, Roma, Antonio Stango Editore, 2003.

Guido Mazzoni, Sulla poesia moderna, Bologna, il Mulino, 2005.

Guido Mazzoni, Sulla storia sociale della poesia contemporanea in Italia, in «Ticontre. Teoria Testo Traduzione», 8 (2017), pp. 1-26.

Francesco Perardi, La forza centripeta conduce l'universo, in «L'Indice dei libri del mese», 6 (2021), p. 38.

Alberto Russo Previtali, Jean Nimis (a cura di), L'avventura della permanenza. La poesia di Milo De Angelis, Milano-Udine, Mimesis, 2020. 
Jean Starobinski, Il concetto di nostalgia, trad. it. Alessandro Serra, in Nostalgia. Storia di un sentimento, a cura di Antonio Prete, Milano, Raffaello Cortina, 1992 (ed. or. Paris 1966).

Gianluigi Simonetti, La letteratura circostante. Narrativa e poesia nell'Italia contemporanea, Bologna, il Mulino, 2018.

Luigi Tassoni, Il gioco infinito della poesia. La lettura dei componimenti da Ungaretti a De Angelis, Roma, Giulio Perrone Editore, 2021.

Pier Vittorio Tondelli, Un weekend postmoderno: cronache dagli anni Ottanta, Milano, Bompiani, 1990.

Gianni Vattimo, Le avventure della differenza, Milano, Garzanti, 1980.

Gianni Vattimo, La fine della modernità, Milano, Garzanti, 1985.

Marco Villa, La sintassi di Somiglianze. Sulla poesia di Milo De Angelis, Pisa, Pacini, 2019.

Marek Zaleski, Formy pamięci, Gdańsk, słowo/obraz terytoria, 2004.

Andrea Zanzotto, Fosfeni, Milano, Mondadori, 1983.

\section{Sitografia}

Giulia Martini, Gli oggetti poetici degli anni Venti, Intervento al convegno TwentyTwenty Extended Conference: Interpreting 21st century poetry, $23 / 02 / 2021$

<https://www.youtube.com/watch?v=GCf1C65Fdfo\&t=3277s > 